zur Kennzeichnung der Zusammensetzung von Heißwalzenmassen eignet, sondern auch für die Untersuchung von Dachpappen von Wert sein dürfte. Bekanntlich kommen zurzeit vielfach unbesandete Dachpappen in den Handel, welche als Imprägnierungsmasse nicht Steinkohlenteer, sondern. Fettdestillationsrückstände oder Mischungen dieser mit Erdölrückständen enthalten. Das Fehlen von Steinkohlenteer erkennt man mit Sicherheit durch erschöpfendes Ausziehen der Pappe mit Benzol. Die so rom Bitumen befreite Pappe enthält nur bei vorheriger Gegenwart von Steinkohlenteer erhebliche Mengen fein verteilter kohliger Stoffe.

Das ausgezogene Bitumen zeigt bei Vorliegen von Fettpech dessen charakteristischen Geruch und bildet beim Erhitzen Acroleindämpfe, gibt außerdem eine beträchtliche Verseifungszahl. War neben Fettpech auch Erdölrückstand (oder Naturasphalt) zugegen, so tritt außerdem die Quecksilberbromidprobe positiv ein.

[A. 91.]

\section{Vorschlag \\ zu einer maßstäblichen Bemessung der Lichtwirkung auf Farbstoffe nach ,Bleichstunden".}

\author{
Von Dr. P. KraIs in Tübingen.
}

(Eingeg. 2.6. 1911.)

Über die Versuche, die gemacht worden sind, um leicht handliche, objektiv zuverlässige MeBinstrumente und Meßverfahren auszubilden, die zu einer Bestimmung der chemischen Wirksamkeit des Lichtes dienen können, findet man Zusammenstellungen bei $J$. W i e s n e r : ,Der Lichtgenu $\beta$ der Pflanzen" (W. Engelmann, Leipzig, 1907), und in E. R übels Arbeiten über das photochemische Klima verschiedener Gegenden (Vierteljahrsschrift der naturforschenden Gesellschaft in. Zürich, Jahrg. 53 [1908], 4 [1909], und 55 [1910]). Es handelt sich hierbei in erster Linie um periodische Einzelbestimmungen der jeweiligen chemischen Intensität des Lichtes.

Über die spezifische Einwirkung der verschie. denen Strahlenarten des Lichtes hat $K$. G e b$h$ a $\mathrm{rd}$ Untersuchungen angestellt ( $\mathrm{L}$ e $\mathrm{h}$ n es Färber-Ztg. 1911, 28).

Im Jahre 1894 hat ein englisches Komitee eine Skala von Färbungen aufgestellt, nach deren Echtheitsverschiedenheiten die Lichtechtheit in fünf Grade eingeteilt werden sollte ( $J$. Soc. Chem. Ind. $1894,803 ; 1896,798)$, aber diese Skala hat sich nicht in der Praxis einführen können. Neuerdings wird in englischen Veröffentlichungen wieder auf sie zurückgegriffen (ebenda 1911, 6 u. 96).

Im Jahre 1900 ist die Ind ustrielle Ge sellschaft in $M \ddot{u} l$ hausen i. Els. bemüht gewesen, eine maßstäbliche Bemessung der Lichtwirkung auf Farbstoffe auszuarbeiten (Bll. Juli bis August, S. 20lff.), ohne aber zu einem befriedigenden Resultat zu gelangen. E m i 1 B e c h t e l hat dann im Jahre 1909 eine aktinometrische Methode vorgeschlagen (Rev. mat. col. 14\%, 73 [1909]; diese Z. 22, 1038), die auf der von $\mathrm{M}$ a $\mathrm{l}$ u s c h e $\mathrm{k}$ (Chem.
Ztg. 1901, 411) beobachteten Zersetzung von Ferround Ferricyaniden in wässeriger Lösung am Licht beruht. Diese Zersetzung läßt sich aber deshalb nicht als Maßstab für die Lichtwirkung benutzen, weil, wie $\mathrm{M}$ a l u s che k ganz klar ausspricht, die Menge des durch das Licht ausgeschiedenen Ferrihydroxyds in arithmetischer Reihe zunimmt, wenn die Konzentration der lösung in arithmetischer Reihe abnimmt.

Neuerdings hat $P$ a u 1 D o s ne (Rev, mat. col. 1910 , 194) vorgeschlagen, die Lichtwirkung mittels des Aktinometers von $\mathrm{Bess}$ on \& $\mathrm{Th}$ u $\mathrm{r}$. e y s s e n zu bemessen. Dieser Apparat beruht auf der Messung des Äthers, der in einem evakuierten Glasgefäß bei Bestrahlung durch die Sonne ver. dunstet. Dos n e beobachtet aber ganz richtig, daß auch bei zerstreutem Tageslicht eine Bleichwirkung auf die Farbstoffe stattfindet, dabei aber das Aktinometer nicht arbeitet. Er verlangt deshall, man solle, wenn die Sonne nicht scheint, die Belichtungsproben zudecken. Daß ein-solches Ver. fahren in der Praxis nicht durchführbar ist, selbst wenn der Apparat durch seine geistreiche Konstruktion noch so verlockend erscheint, bedarf wohl keiner weiteren Begründung.

Einen für die Praxis wertvollen Vorschlag hat Ed u a r V V le n t a gemacht (Chem.-Ztg. 1906, 901 und 1909, 1165), indem er für die Lichtechtheit von Buchdruckfarben den Alizarinrotlack als Typ aufstellte, seine Echtheit $=1000$ setzte und die anderen Farben nach diesem Typ bemaß. Dieses Verfahren wird sich wohl vielfach eingebürgert haben und wird auch neuerdings von $\mathrm{A}$. $\mathrm{F}$ i b n e $\mathrm{r}$ (Farbenzeitung 1911. 1815) als die für praktische Zweeke noch am besten geeignete Methode empfohlen. Immer aber haftet ihr der Mangel an, daß ein willkürlich gewählter Farbstoff als Typ aufgestellt wurde, und daß sie nicht von allgemeiner Anwendbarkeit ist.

Diese kurze Zusammenstellung, die auf Vollständigkeit keinen Anspruch machen will, zeigt zur Genüge, daB es uns heute noch an einem befriedigenden Maßstab für die Bemessung der Lichtwirkung auf Farbstoffe fehlt. Ein weiterer Beweis hierfür sind ja die nach Richtung und Prinzip so verschiedenen und oft ganz phantastischen Echtheitsbezeichnungen und normierungen, wie sie in der Industrie der Farben (im weitesten Sinne) gang und gäbe sind.

Für genaue Bestimmungen der Tonabschwächung und Nuancenveränderung der Farben durch das Licht gibt es heute eine ganz respektable Anzahl von Tintometern, Colorimetern, Farbenanalysatoren und Chromoskopen und wie die Apparate alle heißen. Obwohl auch hier Einheitlichkeit zu erstreben ist, wird diese Frage erst dann wichtig, wenn ein einheitlicher Maßstab für die L i c h twirkung gefunden und akzeptiert worden ist.

Erst dann kann die jetzige durch alle mit Farben arbeitenden Produzenten- wie Konsumentenkreise gehende Unsicherheit und Unklarheit ausgeschaltet werden.

Es ist selbstverständlich, daß ein solcher Maßstab in erster Linie zuverlässig und allgemein anwendbar, in zweiter Linie einfach in der Handhabung sein muß. Komplizierte Apparate, die man 
fortwh hread kontrollieren $\mathrm{muB}$, and bei denen es immer wieder Reparaturen gibt, werden sich nioht einfüihren.

Es war daher während der letzten Monate mein Bestreben, einen möglichst einfachen und verständlichen Weg einzuschlagen, und dieser Weg besteht darin, die bleichende $K r$ aft der Son ne auf einen rasch verbleichenden Farbstoff zum Maßstab zu wählen. Ich mache keinen Anspruch darauf, daB dies etwa ein neuer Gedanke sein soll, aber ich weiß, daß er bis jetzt nicht in einer Weise ausgebeutet worden ist, die zu einem allgemein angenommenen Maßstab geführt hat.

Um eine gleichmäßig gefärbte Schicht zu erzeugen, wähle ich einen rasch verbleichenden Farbstoff, der auf einem weißen Substrat niedergeschlagen ist, und trage diesen nach Art der Tapetenfarben auf Papier auf. Als Bleichmittel wähle ich die hochstehende Sonne bei klarem Himmel im Freien.

Die Anwendung des Maßstabes ist nun so einfach wie die von Reagenspapieren (auch diese müssen ja richtig zubereitet sein), er ist mit gleicher Zuverlässigkeit für zerstreutes Tageslicht, für künstliches Licht, für offenes Licht oder hinter Glas zu gebrauchen, sobald die „Bleichstunde" festgestellt ist. -

Es ist bekannt, daB basische Farbstoffe, wie Methylviolett, Malachitgrün, Viktoriablau sich in der Kälte auf allerhand Erden niederschlagen lassen, und daß die so entstehenden Farben, so schön sie sind, äußerst lichtempfindlich sind, insbesondere, wenn weiße Erden als Substrat dienen.

Einige Vorversuche zeigten, daß von den vorgenannten drei Farbstoffen das Viktoriablau nach mehratündiger Belichtung seinen Charakter als Blau noch am besten bewahrt hatte, während Malachitgriin und Methylviolett mehr nach Grau verschießen, wodurch die Abschätzung des Bleichgrades erschwert wird.

Es wurde also eine Farbe hergestellt, die sich fuir meinen Zweck bisher als geeignet erwiesen hat, und die zusammengesetzt ist, wie folgt:

$200 \mathrm{~g}$ weißes Kaolin (Porzellanerde, China Clay) wurden mit $200 \mathrm{ccm}$ Wasser angeteigt und hierzu $400 \mathrm{ccm}$ einer Lösung von genau I g Viktoriablau $\mathrm{R}$ (A.-G. für Anilinfabrikation, Berlin) in $1000 \mathrm{ccm}$ dest. Wasser gegeben. Nach gutem Durchrühren des Gemisches wurde dieses abgesaugt (das Filtrat ist ganz farblos), die Farbe bei gewöhnlicher Temperatur im Dunkeln getrocknet, dann zerrieben. Von dem trockenen Farbstoff wurden $50 \mathrm{~g}$. mit $35 \mathrm{ccm}$ Wasser angerührt und dann $45 \mathrm{ccm}$ einer Lösung von $50 \mathrm{~g}$ Gummi arabicum in $250 \mathrm{ccm}$ Wasser (die vorher durch ein feines Leintuch passiert war) zugegeben. Die nun fertige Streichfarbe wurde mit einem Pinsel auf holzfreies, ungeleimtes Papier (Nr. 465 der Papierfabrik Scheufelen in OberlenningenTeck) aufgetragen, mit einem sog. Vertreiberpinsel geglättet und an der Luft getrocknet. Die Farbe reicht für etwa 14 Bogen von $22 / 28 \mathrm{~cm}$ Größe, und die Bogen halten sich im Dunkein und trocken aufbewahrt beliebig lange.

Die mit diesem „Belichtungspapier" angestellten weiteren Versuche zeigten nun

1. daß man im Sonnenlicht von Stunde zu Stunde eine deutlich abgestufte Verbleichung er- hält, und dá im Mal die blelchwrknng pro stumde in der Zeit von $10 \mathrm{Uhr}$ morgens bis $4 \mathrm{Uhr}$ rachmittags annähernd gleioh war,

2. daB die Intervalle innerhalb der ersten sechs Belichtungsstunden deutlich zu unterscheiden sind, während sie dann undeutlich werden, weil fast kein Farbstoff mehr vorhanden ist,

3. daB die Verbleichung im offenen Sonnenlicht etwas, aber nicht viel stärker ist, als hinter $6 \mathrm{~mm}$ dickem Spiegelglas. Deutlich wird der Untersehied in der Verbleichung erst, wenn die Sonne in ziemlich schräger Richtung auf das Glas scheint, offenbar, weil dann ein Teil der Strahlen reflektiert wird,

4. daB die Bleichproben sich nicht etwa nach einiger Zeit der Aufbewahrung im Dunkeln wieder ,erholen", wie man das bei manchen Malachitgrünlacken beobachten kann, sondern daß die einmal eingetretene Verbleichung unverändert bleibt,

5. $\mathrm{da} B$ es, um ein einwandfreies Bild für die Vergleichung mit dem MaBstab zu bekommen, empfehlenswert ist, das Papier nicht länger als fünf Stunden der offenen Sonne auszusetzen.

\section{Die Herstellungdes MaBstabes.}

Die Herstellung des Maßstabes gestaltet sich sehr einfach. Man benutzt klare Sonnenstunden, vorzugsweise zwischen 11 und $3 \mathrm{Uhr}$, und belichtet die eine Serie von Proben immer je eine Stunde, eine andere 1, 2, 3, 4 und 5 Stunden lang. Die erste Serie dient zur Kontrolle dafür, daß die Bleichwirkung in allen fünf Stunden relativ gleich war, die zweite Serie bildet den Maßstab.

Der Gebrauch des MaBstabes ist ebenso einfach. Man exponiert in der Belichtungsstation jeden Tag einen frischen Streifen des Blaupapieres, an sehr sonnigen Tagen muß man natürlich um die Mittagszeit nochmals wechseln, sammelt die Streifen, auf denen man Tag und Zeit oder Nummer vermerkt hat, und liest dann am Ende einer Belichtungsperiode die "Bleichstunden" durch Vergleichung mit dem Maßstab ab, die man dann addiert, um die gesamte Bleichwirkung durch die Anzahl der Bleichstunden ausdrücken zu können.

Um die Belichtung für die Herstellung des MaBstabes möglichst gleichmäßig und intensiv $\mathrm{zu}$ gestalten, habe ich die Papierstreifen auf ein Brett gespannt und dieses auf das Objektiv eines größeren Fernrohres gebunden. Mit dem am Fernrohr befindlichen Sucher, dessen Okular geschwärzt war, wurde dann die Belichtungsfläche alle 5-10 $\mathrm{Min}$. auf die Sonne eingestellt, so da $B$ die Sonnenstrahlen immer fast genau senkrecht auf das Papier fielen.

Aus der folgenden Tabelle geht hervor, daB die Bemessung nacb Sonnenstunden keinen genügend genauen Anhalt für die Belichtungswirkung gibt. Die Sonnenstunden wurden mit einem Autographen (C a m p b e I l - S t o k es) gemessen.

Während in der Zeit vom 10.-18./5. auf 24,5 Sonnenstunden 31 ,Bleichstunden" kamen, zeigt die Zeit vom 19.-27. trotz der gemessenen 54 Sonnenstunden nur 35,5 Bleichstunden. Dieg kommt daher, daß das zerstreute Tageslicht auch bleichend wirkt, und $\mathrm{da} \beta$ an den sehr sonnigen Tagen vom 21., 22., 26. und 27./5. die Sonne nur in den mittleren Tagesstunden die volle Bleichwirkung ausüben konnte, weil die Belichtungsproben, die 
Mai 1911 Sonnenstunden „Bleichstunden" Papier.N.

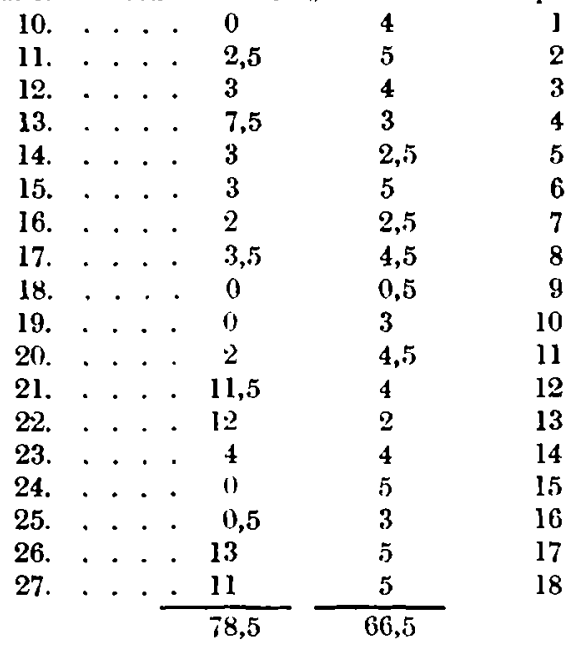

sich in diesem Falle hinter eiver $6 \mathrm{~mm}$ dicken Spiegelscheibe befanden, nach Süden gerichtet waren.

Der neue MaBstab nach Bleichstunden sollte sich leicht einführen lassen und würde wesentlich zu einer Vereinheitlichung auf diesem Gebiete beitragen. Ich bin gern bereit, an Kollegen einige Bogen des Blaupapieres abzugebon. Sollte mein Vorschlag Anklang finden, so wäre es cin Leichtes, etwa bei einer Tupetenfabrik einigo Rollen Papier mit der Farbe streichen zu Iassen, die dann als Vorrat für eine Reihe von Jahren und für viele Belichtungsstationen ein einheitliches Material bilden würden. Ich bin bereit, die hicrmit verbundene Mïhe und Verantwortung zu übernebmen und auch eine Anzahl von Maßstäben in gleichartiger Weise herzustellen, vorausgesetzt, da $B$ etwa die Fachgruppe die Frage der Herstellungskosten und des etwaigen Verkaufspreises des MaßBstabes regelt.

Finige Korrekturen wären für dic allgenuine Benutzung des Maßstabes wohl noch zu berücksichtigen, abgesehen von Vorschlägen, die mir otwa noch von seiten der Kollegen zugehen. Vor allem würden die Bleichstunden für den Maßstab nicht schon um 10 Uhr morgens $7 u$ beginnen sein, denn die am 26. $/ 5$. aufgenommene erste Bleichstunde ist etwas schwächer, als die späteren. Ferner würde man überhaupt in erster Linie die Stunden des höchsten Sonnenstandes im Hochsommer benutzen müssen.

$$
\text { Nachtrag rom 35. Juni } 1911 .
$$

Inzwischen ist es mir gelungen, eincn Maßstab über fünfeinhalb Stunden mit halbstündigen Intervallen bei ganz klarer Sonne in den Mittagsstunden herzustellen, der eine ganz regelmäßige Abstufung zeigt. Ich habe ein Exemplar dieses Maßstabs an den neugewählten Vorsitzenden unserer Farhgruppe und der Ech theitskommission, Herrn Geheimrat Dr. Lehne eingesandt.

Fin wesentlicher Vor\%ug, den meine Methode hat, scheint. mir zu sein, daB aie infolge ihrer Einfachheit und leichten Anwendbarkeit ein weites Versuchsfeld eröfnet, weil keine Apparate und keine langwierigen analytirchen Bestimmungen nötig sind, während Benbachtungsfohler so gut wie ausge. schlossen erscheinen. Es können also z. B. mit geringer Mühe nachgeprüft werden:

I. die aktinische Wirkung verschiedener Lichtquellen und Lichtarten.

2. die Verschiedenheiten in der Bleichwirkung bei verschiedenen Feuchtigkeitsverhältnissen.

Gegenwärtigr bin ich mit der für die Praxis sehr wichtigen Frage beschäftigt. wie die Bleichwirkung sich in den vier Himmelsrichtungen und im Zimmer, im Frcien, an der Decke, am Fenster usw. differenziert.

Interessante Aufschlüsse verspreche ich mir auch von einer Untersuchung der reflektierenden Wirkung verschiedener, insbesondeje weißer Körper, weil sie auf die immer noch nicht zu aller Befriedigung grelöste Frage der Deckwirkung der weiben Farben und Substrate Iicht werfen könnte.

Auch für den Botaniker und Pflanzenphysiologen, für den Geographen und Klimatologen sollte meine Methode Interesse hahen, denn wenn der .Maßstab" einmal festgelegt ist, hat er seine Borochtigung so gut wie jeder andere, wio Thermo. meter und Barometer.

P. Krais.

\section{Geschichtliches über die Cellulose- acetate.}

\author{
Von H. OsT-Hannover.
}

(Eingeg. 11.5. 1911. )

In einem am 21./12. 1910 in Berlin gehaltenen Vortrage sagt $\mathbf{E}$ i che $n g$ r ün u. a., er habe bereits 1901 als erster den Nachwris geführt, da 3 die chloroformlöslichen Celluleseacetate nicht 'Tetra-, sondern Triacetate seien, und dieser Befund sei von mir und meinen Schülern später bestätigt worden ${ }^{\mathbf{1}}$ ). Da die Celluloseacetate inzwischen eine wichtige Er. findung geworden sind, so halte ich es für nötig, diese Angaben $\mathrm{E}$ i c he $\mathrm{n} g \mathrm{r}$ ï $\mathrm{n}$ s durch einige geschichtliche Notizen zu berichtigen und zu ergänzen. Die Erfinder technisch brauchbarer Celluloseacetate sind ('ross und Bevan, deren Darstellungsne. thoden aus Cellulose (mercerisierter oder nicht mercerisierter) mittels (hloracetyl und Zink-oder Magnesiumacetat bei niedriger Temperatur, nach D. R. P. 85329 (1894) und 86.363 (1895) wehr unbequem und unsicher waren. Einen wesentlichen Fortschritt brachte Lederer mit D. R. P'. 1185,38 (1899) und l:20 713 (l90()), welcher von den Hydrocellulosen $\mathrm{G}$ i $\mathrm{r}$ a $\mathrm{rd}$ s aukging und diese mit. Essig. säureanhydrid und wenig konz. Schwefelsäure bci niedriger Temperatur leicht und glatt acetylierte. Beide Erfinder haben aber ihre in Chloroform löslichen Acetate irrtümlich für Tetracetal

$$
\mathrm{C}_{6} \mathrm{H}_{6} \mathrm{O}_{5}\left(\mathrm{C}_{2} \mathrm{H}_{3} \mathrm{O}\right)_{4} \text {, }
$$

bzw. für Gemenge von Tetra- und Triacetat gehalten, infolge ihrer irrefülırenden Verseifung der Acetate anit kochendem Alkali, wodurch Cellulose selbst etwas Sïure bildet, die sie zusammen mit der Essigsäure titrierten.

Unter deul 2./8. 1901 meldeten die Farbenfabriken Fr. Bayer \& Co. das später erteilte

1) Diese Z. 24,366 (1911). 